International Journal of Algebra, Vol. 7, 2013, no. 2, 91 - 100 HIKARI Ltd, www.m-hikari.com

\title{
A Sequence of Weakly Monotonic Automata with Increasing Level
}

\author{
Jorge Almeida and Ângela Cardoso \\ CMUP, Departamento de Matemática \\ Faculdade de Ciências, Universidade do Porto \\ Rua do Campo Alegre, 687 \\ 4169-007 Porto, Portugal \\ jalmeida@fc.up.pt \\ angela.cardoso@fc.up.pt
}

Copyright (c) 2013 Jorge Almeida and Ângela Cardoso. This is an open access article distributed under the Creative Commons Attribution License, which permits unrestricted use, distribution, and reproduction in any medium, provided the original work is properly cited.

\begin{abstract}
In this paper, we exhibit a sequence of strongly connected aperiodic weakly monotonic automata, whose level increases with the number of states.
\end{abstract}

Mathematics Subject Classification: 20M35, 68Q45, 20M07

Keywords: Weakly monotonic automata, aperiodic automata, strongly connected automata

\section{Introduction}

Forty five years ago, Černý [1] presented a family of synchronizing automata with $n$ states whose shortest reset words have length $(n-1)^{2}$ and conjectured that for every automaton with $n$ states, if there is a synchronizing word, then there is one with at most $(n-1)^{2}$ letters. Several advances have been made towards the proof of this conjecture, but the general case remains open. For more information on the history of the Cerný Conjecture and the particular cases that have been solved, see for example [3]. 
The class of aperiodic automata has received some attention recently regarding its synchronizing properties. Particularly, in [2] Trahtman established the Černý conjecture for this class of automata, proving that every synchronizing aperiodic automaton with $n$ states has a reset word whose length is at most $n(n-1) / 2$. Later in [4], Volkov introduced the concept of weakly monotonic automaton and proved that every aperiodic automaton is weakly monotonic. Then he established that every synchronizing strongly connected weakly monotonic automaton has a reset word whose length is not greater than $\lfloor n(n-1) / 6\rfloor$. This way, Trahtman's bound was improved for the case of strongly connected aperiodic automata.

In this paper, we show that given $l \in \mathbb{N}$, there is a synchronizing strongly connected aperiodic automaton that is weakly monotonic of level $l$. The reason to search for strongly connected and aperiodic automata is the fact that in his paper Volkov suspects that the bound $\lfloor n(n-1) / 6\rfloor$ can be further improved for this particular case. In private communication, Volkov asked the second author whether there is a bound on the level of weak monotonicity of strongly connected aperiodic automata, in the hope that such a property would entail fast synchronization.

\section{Definitions}

Given a complete deterministic finite automaton $\mathcal{A}=(S, \Sigma, \delta)$, a binary relation $\rho \subset S \times S$ in $\mathcal{A}$ is stable if for every $\sigma \in \Sigma$ and every $p, q \in S,(p, q) \in \rho$ implies $(\delta(p, \sigma), \delta(q, \sigma)) \in \rho$. The equivalence closure of a binary relation $\rho$, denoted by $\operatorname{Eq}(\rho)$, is the smallest equivalence relation that contains $\rho$. Of course, if $\rho$ is stable so is $\operatorname{Eq}(\rho)$. A congruence over $\mathcal{A}$ is a stable equivalence relation $\pi \subset S \times S$.

Given a congruence $\pi$, denote by $[p]_{\pi}$ the $\pi$-class that contains the state $p \in S$. One defines the quotient automaton $\mathcal{A} / \pi$ as the automaton $\left(S / \pi, \Sigma, \delta_{\pi}\right)$, where $S / \pi=\left\{[p]_{\pi}: p \in S\right\}$ and the transition function $\delta_{\pi}$ is such that for every $\pi$-class $[p]_{\pi}$ and every $\sigma \in \Sigma, \delta_{\pi}\left([p]_{\pi}, \sigma\right)=[\delta(p, \sigma)]_{\pi}$.

The complete deterministic finite automaton $\mathcal{A}=(S, \Sigma, \delta)$ is said to be weakly monotonic of level $l$, see [4], if there is a strictly increasing chain of stable binary relations $\rho^{0} \subset \rho^{1} \subset \ldots \subset \rho^{l}$ on $\mathcal{A}$ such that:

- $\rho^{0}$ is the equality relation $\{(s, s): s \in S\}$;

- for each $i \in\{1,2, \ldots, l\}, \pi^{i-1}=\operatorname{Eq}\left(\rho^{i-1}\right) \subset \rho^{i}$ and $\rho^{i} / \pi^{i-1}$ is a partial order on $S / \pi^{i-1}$;

- $\pi^{l}=\operatorname{Eq}\left(\rho^{l}\right)$ is the universal relation on $S$.

A semigroup $A$ is said to be aperiodic if all its subgroups are trivial, which is equivalent to the property that for every $a \in A$, there is some $m \in \mathbb{N}$ such 
that $a^{m}=a^{m+1}$. An automaton $\mathcal{A}=(S, \Sigma, \delta)$ whose transition semigroup is aperiodic is called aperiodic as well.

We say that the automaton $\mathcal{A}=(S, \Sigma, \delta)$ is strongly connected if its underlying digraph $G$ is strongly connected, that is, for all $p, q \in S$ there is a directed path in $G$ going from $p$ to $q$.

\section{A Sequence Of Automata}

For each positive integer $n$, consider the complete deterministic finite automaton $\mathcal{A}_{n}=\left(S_{n}, \Sigma_{n}, \delta_{n}\right)$, with set of states $S_{n}=\{0,1, \ldots, n-1\}$, alphabet $\Sigma_{n}=\left\{\sigma_{0}, \sigma_{1}, \ldots, \sigma_{n-1}\right\}$ and transition function $\delta_{n}$ such that:

- for each $i \in 0,1, \ldots, n-2$ and each $s \in S$,

$$
\delta_{n}\left(s, \sigma_{i}\right)=\left\{\begin{array}{ll}
i & \text { if } s<n-i-1 \text { or } s=n-i-1 \geq\left\lceil\frac{n}{2}\right\rceil \\
i+1 & \text { if } s \geq n-i \text { or } s=n-i-1<\left\lceil\frac{n}{2}\right\rceil
\end{array} ;\right.
$$

- for every $s \in S$,

$$
\delta_{n}\left(s, \sigma_{n-1}\right)=\left\{\begin{array}{ll}
n-s-1 & \text { if } s<\left\lceil\frac{n}{2}\right\rceil \\
n-s & \text { if } s \geq\left\lceil\frac{n}{2}\right\rceil
\end{array} .\right.
$$

Note that

for every $n>1$ and every $\sigma \in \Sigma_{n}, \delta_{n}\left(\left\lceil\frac{n}{2}\right\rceil, \sigma\right)=\delta_{n}\left(\left\lceil\frac{n}{2}\right\rceil-1, \sigma\right)$.

To represent the automaton $\mathcal{A}_{n}$ we use a $n \times n$ matrix over $\mathbb{N}$ such that for $i, j \in\{0,1, \ldots, n-1\}$ the entry $(i, j)$ is $\delta_{n}\left(i, \sigma_{j}\right)$.

Example 3.1. The matrices that represent the automata $\mathcal{A}_{1}, \mathcal{A}_{2}, \mathcal{A}_{3}, \mathcal{A}_{4}$ and $\mathcal{A}_{5}$ are, respectively:

$$
(0),\left(\begin{array}{ll}
0 & 1 \\
0 & 1
\end{array}\right),\left(\begin{array}{lll}
0 & 1 & 2 \\
0 & 2 & 1 \\
0 & 2 & 1
\end{array}\right),\left(\begin{array}{llll}
0 & 1 & 2 & 3 \\
0 & 1 & 3 & 2 \\
0 & 1 & 3 & 2 \\
0 & 2 & 3 & 1
\end{array}\right) \text { and }\left(\begin{array}{lllll}
0 & 1 & 2 & 3 & 4 \\
0 & 1 & 2 & 4 & 3 \\
0 & 1 & 3 & 4 & 2 \\
0 & 1 & 3 & 4 & 2 \\
0 & 2 & 3 & 4 & 1
\end{array}\right) .
$$

Lemma 3.2. Let $\rho$ be a stable and transitive binary relation on $\mathcal{A}_{n}$. Suppose that $(s, t) \in \rho$ with $s \neq t$ and that there is a nonempty set $I_{t}^{s}$ of consecutive elements of $\{0,1, \ldots, n-2\}$ such that for each $i \in I_{t}^{s}, \delta_{n}\left(s, \sigma_{i}\right)=i$ and $\delta_{n}\left(t, \sigma_{i}\right)=i+1$. Suppose also that for $p=\min I_{t}^{s}$ and $q=\max I_{t}^{s}+1$, $\delta_{n}\left(s, \sigma_{n-1}\right)=q$ and $\delta_{n}\left(t, \sigma_{n-1}\right)=p$. Then the relation $\rho$ cannot be antisymmetric. 
Proof. We know that for every $i \in I_{t}^{s}, \delta_{n}\left(s, \sigma_{i}\right)=i$ and $\delta_{n}\left(t, \sigma_{i}\right)=i+1$, therefore

$$
\begin{gathered}
\delta_{n}\left(s, \sigma_{p}\right)=p \text { and } \delta_{n}\left(t, \sigma_{p}\right)=p+1, \\
\delta_{n}\left(s, \sigma_{p}+1\right)=p+1 \text { and } \delta_{n}\left(t, \sigma_{p}+1\right)=p+2, \\
\vdots \\
\delta_{n}\left(s, \sigma_{q-1}\right)=q-1 \text { and } \delta_{n}\left(t, \sigma_{q-1}\right)=q .
\end{gathered}
$$

Since $\rho$ is stable and $(s, t) \in \rho$, we have $(p, p+1),(p+1, p+2), \ldots,(q-1, q) \in \rho$. Using the transitivity of $\rho$, we conclude that $(p, q) \in \rho$. But we also have $(q, p) \in \rho$, because $\delta_{n}\left(s, \sigma_{n-1}\right)=q$ and $\delta_{n}\left(t, \sigma_{n-1}\right)=p$ and $\rho$ is stable. Thus, since $p \neq q$ because $I_{t}^{s}$ is nonempty, $\rho$ cannot be antisymmetric.

Lemma 3.3. Let $\pi_{n}^{0}$ be the equality relation on $\mathcal{A}_{n}$. The only stable partial orders on $\mathcal{A}_{n}$ are $\pi_{n}^{0}, \rho_{n}^{1}=\pi_{n}^{0} \cup\{(\lceil n / 2\rceil-1,\lceil n / 2\rceil)\}$ and $\bar{\rho}_{n}^{1}=\pi_{n}^{0} \cup$ $\{(\lceil n / 2\rceil,\lceil n / 2\rceil-1)\}$.

Proof. Let $\rho$ be a stable and transitive binary relation on $\mathcal{A}_{n}$ and suppose that $(s, t) \in \rho$ with $s \neq t$ and $\{s, t\} \neq\{\lceil n / 2\rceil-1,\lceil n / 2\rceil\}$. We can assume without loss of generality that $s<t$ for the usual order on $\mathbb{N}$, otherwise it would be enough to consider the reverse order of $\rho$. Since $\{s, t\} \neq\{\lceil n / 2\rceil-1,\lceil n / 2\rceil\}$, we have the following possibilities:

1. $s<t<\lceil n / 2\rceil$;

2. $\lceil n / 2\rceil<s<t$

3. $s<\lceil n / 2\rceil<t$

4. $s=\lceil n / 2\rceil<t$

5. $s<\lceil n / 2\rceil=t$.

In case 1 , note that the set $I_{t}^{s}=\{i \in\{0,1, \ldots, n-2\}: n-t-1 \leq i<$ $n-s-1\}$ is nonempty. For each $i \in I_{t}^{s}$, we have $s<n-i-1 \leq t$, hence $\delta_{n}\left(s, \sigma_{i}\right)=i$ and $\delta_{n}\left(t, \sigma_{i}\right)=i+1$. We also have $\delta_{n}\left(s, \sigma_{n-1}\right)=n-s-1$ and $\delta_{n}\left(t, \sigma_{n-1}\right)=n-t-1$. This way we are in the conditions of Lemma 3.2 and $\rho$ cannot be antisymmetric, which means that it is not a partial order.

In case 2 , we put $I_{t}^{s}=\{i \in\{0,1, \ldots, n-2\}: n-t \leq i<n-s\}$, which is again a nonempty set. For each $i \in I_{t}^{s}$, we have $s<n-i \leq t$, hence $\delta_{n}\left(s, \sigma_{i}\right)=i$ and $\delta_{n}\left(t, \sigma_{i}\right)=i+1$. We also have $\delta_{n}\left(s, \sigma_{n-1}\right)=n-s$ and $\delta_{n}\left(t, \sigma_{n-1}\right)=n-t$. This way we are in the conditions of Lemma 3.2 and $\rho$ cannot be antisymmetric, which means that it is not a partial order.

In case $3, s<t-1$ and so the set $I_{t}^{s}=\{i \in\{0,1, \ldots, n-2\}: n-t \leq i<$ $n-s-1\}$ is nonempty. For each $i \in I_{t}^{s}$, we have $s<n-i-1<t$, hence 
$\delta_{n}\left(s, \sigma_{i}\right)=i$ and $\delta_{n}\left(t, \sigma_{i}\right)=i+1$. We also have $\delta_{n}\left(s, \sigma_{n-1}\right)=n-s-1$ and $\delta_{n}\left(t, \sigma_{n-1}\right)=n-t$. This way we are in the conditions of Lemma 3.2 and $\rho$ cannot be antisymmetric, which means that it is not a partial order.

In case 4 , we have $(\lceil n / 2\rceil, t) \in \rho$, with $t>\lceil n / 2\rceil$. Since $\delta_{n}\left(\lceil n / 2\rceil, \sigma_{n-t}\right)=$ $n-t$ and $\delta_{n}\left(t, \sigma_{n-t}\right)=n-t+1$, so that $(n-t, n-t+1) \in \rho$, we fall again in case 1 unless $n$ is even and $t=n / 2+1$, in which case we have $(n-t, n-t+1)=(n / 2-1, n / 2) \in \rho$ and also $(n / 2, n / 2+1) \in \rho$. But $\delta_{n}\left(n / 2, \sigma_{n-1}\right)=n-n / 2=n / 2$ and $\delta_{n}\left(n / 2+1, \sigma_{n-1}\right)=n-(n / 2+1)=n / 2-1$, so that $(n / 2, n / 2-1) \in \rho$, since $\rho$ is stable. This proves that $\rho$ cannot be antisymmetric because both $(n / 2, n / 2-1)$ and $(n / 2-1, n / 2)$ belong to $\rho$.

In case 5 , since $\{s, t\} \neq\{\lceil n / 2\rceil-1,\lceil n / 2\rceil\}$, we must have $s<\lceil n / 2\rceil-1$ and $t=\lceil n / 2\rceil$, with $(s, t) \in \rho$. Since $\delta_{n}\left(s, \sigma_{n-\lceil n / 2\rceil}\right)=\lceil n / 2\rceil$ and $\delta_{n}\left(t, \sigma_{n-\lceil n / 2\rceil}\right)=$ $\lceil n / 2\rceil+1$, we deduce that $(\lceil n / 2\rceil,\lceil n / 2\rceil+1) \in \rho$, which falls in case 5 , that we have already treated.

To finish the proof, it is enough to verify that $\rho_{n}^{1}$ is a stable partial order on $\mathcal{A}_{n}$, since $\bar{\rho}_{n}^{1}$ is the reverse order of $\rho_{n}^{1}$. In view of $(1)$, we deduce that $\rho_{n}^{1}$ is stable and it is trivial to check that it is reflexive, transitive and antisymmetric.

Given a positive integer $n$, consider the automaton $\mathcal{B}_{n}=\left(S_{n}, \Sigma_{n} \uplus\{\tau\}, \bar{\delta}_{n}\right)$, where $\bar{\delta}_{n}$ is such that for every $s \in S_{n}$,

$$
\left.\bar{\delta}_{n}\right|_{S_{n} \times \Sigma_{n}}=\delta_{n} \text { and } \bar{\delta}_{n}(s, \tau)=\lceil n / 2\rceil-1 .
$$

Lemma 3.4. Consider the stable equivalence relation

$$
\pi_{n}^{1}=\pi_{n}^{0} \cup\{(\lceil n / 2\rceil-1,\lceil n / 2\rceil),(\lceil n / 2\rceil,\lceil n / 2\rceil-1)\}
$$

on $\mathcal{A}_{n}$. Then, for every $n \geq 2$,

$$
\mathcal{A}_{n} / \pi_{n}^{1} \simeq \mathcal{B}_{n-1}
$$

Proof. Consider the functions

$$
\begin{aligned}
& \phi: S_{n} / \pi_{n}^{1} \longrightarrow S_{n-1} \psi: \Sigma_{n} \longrightarrow \Sigma_{n-1} \cup\{\tau\} \\
& {[s] \longmapsto\left\{\begin{array} { l l } 
{ s } & { \text { if } s < \lceil \frac { n } { 2 } \rceil } \\
{ s - 1 } & { \text { if } s \geq \lceil \frac { n } { 2 } \rceil }
\end{array} \quad \sigma _ { i } \longmapsto \left\{\begin{array}{ll}
\sigma_{i} & \text { if } i<\left\lceil\frac{n}{2}\right\rceil-1 \\
\tau & \text { if } i=\left\lceil\frac{n}{2}\right\rceil-1 \\
\sigma_{i-1} & \text { if } i>\left\lceil\frac{n}{2}\right\rceil-1
\end{array}\right.\right.}
\end{aligned}
$$

Note that $\phi$ is well defined, because the only nontrivial class is $\left\{\left\lceil\frac{n}{2}\right\rceil-1,\left\lceil\frac{n}{2}\right\rceil\right\}$. For the same reason, $\phi$ is bijective and it is obvious that $\psi$ is also a bijection. Hence, to finish the proof all we need to check is that the pair $(\phi, \psi)$ defines a morphism between the automata $\mathcal{A}_{n} / \pi_{n}^{1}$ and $\mathcal{B}_{n-1}$, that is, for every $[s] \in$ $S_{n} / \pi_{n}^{1}$ and every $\sigma_{i} \in \Sigma_{n}$

$$
\bar{\delta}_{n-1}\left(\phi([s]), \psi\left(\sigma_{i}\right)\right)=\phi\left(\delta_{n}\left([s], \sigma_{i}\right)\right) .
$$

We have the following possibilities: 
1. $i=\lceil n / 2\rceil-1$

2. $i<\lceil n / 2\rceil-1$ and $s \leq\lceil n / 2\rceil$;

3. $i<\lceil n / 2\rceil-1$ and $s>\lceil n / 2\rceil$;

4. $i>\lceil n / 2\rceil-1$ and $s \leq\lceil n / 2\rceil$;

5. $i>\lceil n / 2\rceil-1$ and $s>\lceil n / 2\rceil$.

In case $1, \psi\left(\sigma_{i}\right)=\tau$ and $\bar{\delta}_{n-1}(t, \tau)=\lceil n / 2\rceil-1$, for all $t \in S_{n-1}$, hence

$$
\bar{\delta}_{n-1}\left(\phi([s]), \psi\left(\sigma_{i}\right)\right)=\left\lceil\frac{n}{2}\right\rceil-1, \text { for every }[s] \in S_{n} / \pi_{n}^{1} .
$$

On the other hand,

$$
\delta_{n}\left([s], \sigma_{i}\right)= \begin{cases}{[i]} & \text { if } s<n-i-1 \text { or } s=n-i-1 \geq\lceil n / 2\rceil \\ {[i+1]} & \text { if } s \geq n-i \text { or } s=n-i-1<\lceil n / 2\rceil\end{cases}
$$

Since $[i]=\{i, i+1\}=[i+1]$ and $\phi([i])=\lceil n / 2\rceil-1$, we have

$$
\phi\left(\delta_{n}\left([s], \sigma_{i}\right)\right)=\left\lceil\frac{n}{2}\right\rceil-1, \text { for every }[s] \in S_{n} / \pi_{n}^{1} .
$$

Therefore, the equality (2) holds in this case.

In case $2, \psi\left(\sigma_{i}\right)=\sigma_{i}$ and $\phi([s])=s$, for $s<\lceil n / 2\rceil$. For $s=\lceil n / 2\rceil,[s]=$ $[\lceil n / 2\rceil-1]$, so it is enough to consider $s<\lceil n / 2\rceil$. It follows that

$$
\begin{gathered}
\bar{\delta}_{n-1}\left(\phi([s]), \psi\left(\sigma_{i}\right)\right)=\bar{\delta}_{n-1}\left(s, \sigma_{i}\right)=\delta_{n-1}\left(s, \sigma_{i}\right)= \\
=\left\{\begin{array}{ll}
i & \text { if } s<n-i-2 \text { or } s=n-i-2 \geq\left\lceil\frac{n-1}{2}\right\rceil \\
i+1 & \text { if } s \geq n-i-1 \text { or } s=n-i-2<\left\lceil\frac{n-1}{2}\right\rceil
\end{array} .\right.
\end{gathered}
$$

But $i<\lceil n / 2\rceil-1$ implies $n-i-2 \geq\lceil(n-1) / 2\rceil$, therefore the condition $s=n-i-2<\lceil(n-1) / 2\rceil$ is impossible and

$$
\bar{\delta}_{n-1}\left(\phi([s]), \psi\left(\sigma_{i}\right)\right)=\left\{\begin{array}{ll}
i & \text { if } s<n-i-1 \\
i+1 & \text { if } s \geq n-i-1
\end{array} .\right.
$$

On the other hand,

$$
\phi\left(\delta_{n}\left([s], \sigma_{i}\right)\right)=\left\{\begin{array}{ll}
\phi([i]) & \text { if } s<n-i-1 \\
\phi([i+1]) & \text { if } s \geq n-i-1
\end{array}=\left\{\begin{array}{ll}
i & \text { if } s<n-i-1 \\
i+1 & \text { if } s \geq n-i-1
\end{array},\right.\right.
$$

because

$$
i<\left\lceil\frac{n}{2}\right\rceil-1 \text { implies }(\phi([i])=i \text { and } \phi([i+1])=i+1) \text {. }
$$


Thus, the equality (2) holds in this case.

Hence

In case $3, \psi\left(\sigma_{i}\right)=\sigma_{i}, \phi([s])=s-1$ and $s-1 \geq\lceil n / 2\rceil \geq\lceil(n-1) / 2\rceil$.

$$
\begin{aligned}
& \bar{\delta}_{n-1}\left(\phi([s]), \psi\left(\sigma_{i}\right)\right)=\bar{\delta}_{n-1}\left(s-1, \sigma_{i}\right)=\delta_{n-1}\left(s-1, \sigma_{i}\right)= \\
& =\left\{\begin{array}{ll}
i & \text { if } s-1<n-i-1 \\
i+1 & \text { if } s-1 \geq n-i-1
\end{array}=\left\{\begin{array}{ll}
i & \text { if } s<n-i \\
i+1 & \text { if } s \geq n-i
\end{array} .\right.\right.
\end{aligned}
$$

On the other hand, in view of (3), we have

$$
\phi\left(\delta_{n}\left([s], \sigma_{i}\right)\right)=\left\{\begin{array}{ll}
\phi([i]) & \text { if } s<n-i \\
\phi([i+1]) & \text { if } s \geq n-i
\end{array}=\left\{\begin{array}{ll}
i & \text { if } s<n-i \\
i+1 & \text { if } s \geq n-i
\end{array} .\right.\right.
$$

Thus, the equality (2) holds in this case.

In case $4, \psi\left(\sigma_{i}\right)=\sigma_{i-1}$ and $\phi([s])=s$, for $s<\lceil n / 2\rceil$. For $s=\lceil n / 2\rceil,[s]=$ $[\lceil n / 2\rceil-1]$, so it is enough to consider $s<\lceil n / 2\rceil$. It follows that

$$
\begin{gathered}
\bar{\delta}_{n-1}\left(\phi([s]), \psi\left(\sigma_{i}\right)\right)=\bar{\delta}_{n-1}\left(s, \sigma_{i-1}\right)=\delta_{n-1}\left(s, \sigma_{i-1}\right)= \\
=\left\{\begin{array}{ll}
i-1 & \text { if } s<n-i-1 \text { or } s=n-i-1 \geq\left\lceil\frac{n-1}{2}\right\rceil \\
i & \text { if } s \geq n-i \text { or } s=n-i-1<\left\lceil\frac{n-1}{2}\right\rceil
\end{array} .\right.
\end{gathered}
$$

But

$$
i>\left\lceil\frac{n}{2}\right\rceil-1 \text { implies } n-i-1<\left\lceil\frac{n-1}{2}\right\rceil \leq\left\lceil\frac{n}{2}\right\rceil,
$$

therefore the condition $s=n-i-1 \geq\lceil(n-1) / 2\rceil$ is impossible and

$$
\bar{\delta}_{n-1}\left(\phi([s]), \psi\left(\sigma_{i}\right)\right)=\left\{\begin{array}{ll}
i-1 & \text { if } s<n-i-1 \\
i & \text { if } s \geq n-i-1
\end{array} .\right.
$$

On the other hand,

$$
\phi\left(\delta_{n}\left([s], \sigma_{i}\right)\right)=\left\{\begin{array}{ll}
\phi([i]) & \text { if } s<n-i-1 \\
\phi([i+1]) & \text { if } s \geq n-i-1
\end{array}= \begin{cases}i-1 & \text { if } s<n-i-1 \\
i & \text { if } s \geq n-i-1\end{cases}\right.
$$

because

$$
i \geq\left\lceil\frac{n}{2}\right\rceil \text { implies }(\phi([i])=i-1 \text { and } \phi([i+1])=i) .
$$

Thus, the equality (2) holds in this case.

Hence

In case $5, \psi\left(\sigma_{i}\right)=\sigma_{i-1}, \phi([s])=s-1$ and $s-1 \geq\lceil n / 2\rceil \geq\lceil(n-1) / 2\rceil$.

$$
\bar{\delta}_{n-1}\left(\phi([s]), \psi\left(\sigma_{i}\right)\right)=\bar{\delta}_{n-1}\left(s-1, \sigma_{i-1}\right)=\delta_{n-1}\left(s-1, \sigma_{i-1}\right)=
$$




$$
=\left\{\begin{array}{ll}
i-1 & \text { if } s-1 \leq n-1-(i-1) \\
i & \text { if } s-1>n-1-(i-1)
\end{array}=\left\{\begin{array}{ll}
i-1 & \text { if } s \leq n-i+1 \\
i & \text { if } s>n-i+1
\end{array} .\right.\right.
$$

On the other hand, in view of (5), we have

$$
\phi\left(\delta_{n}\left([s], \sigma_{i}\right)\right)=\left\{\begin{array}{ll}
\phi([i]) & \text { if } s<n-i \\
\phi([i+1]) & \text { if } s \geq n-i
\end{array}=\left\{\begin{array}{ll}
i-1 & \text { if } s<n-i \\
i & \text { if } s \geq n-i
\end{array} .\right.\right.
$$

Thus, equality (2) holds in this case as well, concluding our proof.

Lemma 3.5. Let $\mathcal{A}=(S, \Sigma, \delta)$ be a complete deterministic finite automaton and consider the automaton $\mathcal{B}=(S, \Sigma \uplus\{\tau\}, \bar{\delta})$, where $\left.\bar{\delta}\right|_{S \times \Sigma}=\delta$ and there is $\bar{s} \in S$ such that, for all $s \in S, \bar{\delta}(s, \tau)=\bar{s}$. Then for every $l \in \mathbb{N}, \mathcal{A}$ is weakly monotonic of level $l$ if and only if $\mathcal{B}$ is weakly monotonic of level $l$.

Proof. Suppose that $\mathcal{A}$ is weakly monotonic of level $l$ for some natural number $l$. Then there is a strictly increasing chain of stable binary relations $\rho^{0} \subset \rho^{1} \subset \cdots \subset \rho^{l}$ on $\mathcal{A}$ such that:

1. $\rho^{0}$ is the equality relation $\{(s, s): s \in S\}$;

2. for each $i \in\{1,2, \ldots, l\}, \pi^{i-1}=\operatorname{Eq}_{\mathcal{A}}\left(\rho^{i-1}\right) \subset \rho^{i}$ and $\rho^{i} / \pi^{i-1}$ is a partial order on $S / \pi^{i-1}$;

3. $\pi^{l}=\operatorname{Eq}_{\mathcal{A}}\left(\rho^{l}\right)$ is the universal relation on $S$.

It is clear that $\rho^{0} \subset \rho^{1} \subset \cdots \subset \rho^{l}$ is a strictly increasing chain of stable binary relations on $\mathcal{B}$, since the state set is the same in both automata, the only letter present in $\mathcal{B}$ that is not in $\mathcal{A}$ acts as a constant, and all these relations contain the equality relation. Trivially, condition 1 holds in $\mathcal{B}$. Condition 2 is also verified, because $\operatorname{Eq}_{\mathcal{A}}(\rho)=\operatorname{Eq}_{\mathcal{B}}(\rho)$ for any binary relation on these automata. Finally, condition 3 holds for the same reason. Which means that $\mathcal{B}$ is weakly monotonic of level $l$. The converse is proved in the same way.

Theorem 3.6. For every positive integer $n$, the automaton $\mathcal{A}_{n}$ is strongly connected, aperiodic and weakly monotonic of level $n-1$.

Proof. It is clear that $\mathcal{A}_{n}$ is strongly connected for every positive integer $n$, because given $s \in S_{n}, \delta_{n}\left(s, \sigma_{0}\right)=0, \delta_{n}\left(0, \sigma_{s}\right)=s$.

Let $T_{n}$ be the transformation monoid of the automaton $\mathcal{A}_{n}$ and consider the associated function $\zeta_{n}: \Sigma_{n}^{*} \longrightarrow T_{n}$. To see that $\mathcal{A}_{n}$ is aperiodic, we will use induction on $n$. The automaton $\mathcal{A}_{1}$ is clearly aperiodic. Moreover, for every idempotent $e \in T_{1}$ and every word $w \in \zeta_{1}^{-1}(e)$, we have $\left|\delta_{1}\left(S_{1}, w\right)\right|=1$, that is $w$ is a synchronizing word. Suppose that, for some integer $n \geq 2, \mathcal{A}_{n-1}$ is aperiodic and, for every idempotent $\bar{e} \in T_{n-1}$ and every word $\bar{w} \in \zeta_{n-1}^{-1}(\bar{e})$, we have $\left|\delta_{n-1}\left(S_{n-1}, \bar{w}\right)\right|=1$. 
Consider the functions

$$
\begin{aligned}
\xi: S_{n} \longrightarrow S_{n-1} & \theta: \Sigma_{n} \longrightarrow \Sigma_{n-1} \\
s & \longmapsto\left\{\begin{array} { l l } 
{ s } & { \text { if } s < \lceil \frac { n } { 2 } \rceil } \\
{ s - 1 } & { \text { if } s \geq \lceil \frac { n } { 2 } \rceil }
\end{array} \quad \sigma _ { i } \longmapsto \left\{\begin{array}{ll}
\sigma_{i} & \text { if } i<\left\lceil\frac{n}{2}\right\rceil-1 \\
\sigma_{0} \sigma_{\lceil n / 2\rceil-1} & \text { if } i=\left\lceil\frac{n}{2}\right\rceil-1 . \\
\sigma_{i-1} & \text { if } i>\left\lceil\frac{n}{2}\right\rceil-1
\end{array}\right.\right.
\end{aligned}
$$

In the proof of Lemma 3.4 we presented an isomorphism $\mathcal{A}_{n} / \pi_{n}^{1} \longrightarrow \mathcal{B}_{n-1}$ that was obtained from functions $\phi: S_{n} / \pi_{n}^{1} \longrightarrow S_{n-1}$ and $\psi: \Sigma_{n} \longrightarrow \Sigma_{n-1} \cup\{\tau\}$, where $\pi_{n}^{1}$ is the kernel of the mapping $\xi$. Thus the pair $(\xi, \theta)$ is obtained by composing the quotient morphism $\mathcal{A}_{n} \longrightarrow \mathcal{A}_{n} / \pi_{n}^{1}$ with the isomorphism $(\phi, \psi): \mathcal{A}_{n} / \pi_{n}^{1} \longrightarrow \mathcal{B}_{n-1}$ and finally with the morphism $\mathcal{B}_{n-1} \longrightarrow \mathcal{A}_{n-1}$ that fixes the states and the letters $\sigma_{i}$ and maps the letter $\tau$ to $\sigma_{0} \sigma_{\lceil n / 2\rceil-1}$. Hence $(\xi, \theta)$ is a morphism of automata. Now, using $\theta$, we can define a morphism $\Theta: T_{n} \longrightarrow T_{n-1}$, by putting the image of an element of $T_{n}$ written as a product of letters in $\Sigma_{n}$, to be the value in $T_{n-1}$ of the product of the images of those letters by $\theta$. All we need to check is that $\Theta$ is well defined, that is, if $\alpha$ and $\beta$ represent the same element of $T_{n}$, then $\Theta(\alpha)$ and $\Theta(\beta)$ represent the same element of $T_{n-1}$. But if $\alpha$ and $\beta$ represent the same element of $T_{n}$, then $\delta_{n}(s, \alpha)=\delta_{n}(s, \beta)$, for for every $s \in S_{n}$. This implies that $\delta_{n-1}(\xi(s), \theta(\alpha))=$ $\delta_{n-1}(\xi(s), \theta(\beta))$, for every $s \in S_{n}$. Or equivalently $\delta_{n-1}(t, \theta(\alpha))=\delta_{n-1}(t, \theta(\beta))$, for every $t \in S_{n-1}$, because $\xi$ is surjective. But this means that $\Theta(\alpha)$ and $\Theta(\beta)$ represent the same element of $T_{n-1}$.

Now, consider an idempotent $e \in T_{n}$, since $\Theta$ is a monoid morphism, we know that $\Theta(e)$ is an idempotent of $T_{n-1}$. Thus, for $w \in \zeta_{n}^{-1}(e)$, we have $\left.\mid \xi\left(\delta_{n}\left(S_{n}\right), w\right)\right)|=| \delta_{n-1}\left(\xi\left(S_{n}\right), \theta(w)\right) \mid=1$, according to the induction hypothesis, because $\theta(w) \in \zeta_{n-1}^{-1}(\Theta(e))$. If $\left|\delta_{n}\left(S_{n}, w\right)\right| \neq 1$, then $\delta_{n}\left(S_{n}, w\right)=\{\lceil n / 2\rceil-$ $1,\lceil n / 2\rceil\}$, because these are the only two distinct elements in $S_{n}$ that have the same image under the function $\xi$. Since $e$ is an idempotent, for all $s \in S_{n}$ we have $\delta_{n}\left(\delta_{n}(s, w), w\right)=\delta_{n}\left(s, w^{2}\right)=\delta_{n}(s, w)$, that is, $w$ fixes all the elements in $\delta_{n}\left(S_{n}, w\right)$. But we know that for every $\sigma \in \Sigma, \delta_{n}(\lceil n / 2\rceil-1, \sigma)=\delta_{n}(\lceil n / 2\rceil, \sigma)$, therefore there is no $w \in \zeta_{n}^{-1}(e)$ such that $\delta_{n}\left(S_{n}, w\right)=\{\lceil n / 2\rceil-1,\lceil n / 2\rceil\}$. Which means that $\left|\delta_{n}\left(S_{n}, w\right)\right|=1$.

We proved that, for every word $w$ in $\Sigma_{n}^{*}$ such that $\zeta_{n}(w)$ is an idempotent of $T_{n}, w$ is a synchronizing word. Now suppose that $x \in T_{n}$ and $m>1$ are such that $x^{m}=x$. Then $x^{m-1}$ is an idempotent, thus every word $w \in \zeta_{n}^{-1}\left(x^{m-1}\right)$ is a synchronizing word in the automaton $\mathcal{A}_{n}$. Consider the word $v \in \zeta_{n}^{-1}(x)$. Then $w v$ is still a synchronizing word and it belongs to $\zeta_{n}^{-1}\left(x^{m-1} x\right)=\zeta_{n}^{-1}\left(x^{m}\right)=$ $\zeta_{n}^{-1}(x)$, therefore $x$ is an idempotent because for each $s \in S_{n}, \delta_{n}\left(s,(w v)^{2}\right)=$ $\delta_{n}(s, w v)$. But this means that if $x^{m}=x$ and $m>1$, then $x^{2}=x$, that is, $T_{n}$ is aperiodic, which means that $\mathcal{A}_{n}$ is aperiodic.

We will also use induction on $n$ to prove that $\mathcal{A}_{n}$ is weakly monotonic of 
level $n-1$. Clearly $\mathcal{A}_{1}$ is weakly monotonic of level 0 . Suppose that for some integer $n \geq 2, \mathcal{A}_{n-1}$ is weakly monotonic of level $n-2$.

According to Lemma 3.3, the only stable partial orders on $\mathcal{A}_{n}$ are the equality relation, $\rho_{n}^{1}=\pi_{n}^{0} \cup\{(\lceil n / 2\rceil-1,\lceil n / 2\rceil)\}$ and $\bar{\rho}_{n}^{1}=\pi_{n}^{0} \cup\{(\lceil n / 2\rceil,\lceil n / 2\rceil-$ 1)\}. Now using Lemmas 3.4 and 3.5, we know that $\mathcal{A}_{n} / \pi_{n}^{1}$ and $\mathcal{A}_{n-1}$ have the same level, where $\pi_{n}^{1}=\operatorname{Eq}\left(\rho_{n}^{1}\right)=\operatorname{Eq}\left(\bar{\rho}_{n}^{1}\right)$. But if the level of $\mathcal{A}_{n} / \pi_{n}^{1}$ is $n-2$ and $\pi_{n}^{1}$ is the only stable equivalence relation obtained from a stable partial order in $\mathcal{A}_{n}$ that is not the equality relation, then $\mathcal{A}_{n}$ is weakly monotonic of level $n-2+1=n-1$.

We have established that for every positive integer $n, \mathcal{A}_{n}$ is a strongly connected aperiodic weakly monotonic automaton of level $n-1$.

We found a family of strongly connected aperiodic automata whose level of weak monotonicity increases with the number of states. Of course, higher levels do not correspond necessarily to bigger reset words. Indeed, adding a letter that acts as a constant function has no effect on the level of weak monotonicity of an automaton, while it makes synchronization quite trivial.

ACKNOWLEDGEMENTS. Research funded by the European Regional Development Fund, through the programme COMPETE, and by the Portuguese Government through FCT - Fundação para a Ciência e a Tecnologia, under the project PEst-C/MAT/UI0144/2011. The second author was supported by the FCT PhD grant SFRH/BD/33099/2007, in the scope of the

programme POPH, financed by the European Social Fund and the Portuguese Government.

\section{References}

[1] J. Černý, Poznámka $k$ homogénnym eksperimentom s konecnými automatami (A note on homogeneous experiments with finite automata), Mat.-Fyz. Cas. Solvensk. Akad. Vied. 14 (1964), 208-216.

[2] A. N. Trahtman, The Černý conjecture for aperiodic automata, Discrete Math. Theor. Comput. Sci. 9 (2007), 3-10 (electronic).

[3] M. V. Volkov, Synchronizing automata and the Černý conjecture, Language and Automata: Theory and Aplications. LATA 2008 (BerlinHeidelberg-N.Y.) (C. Martín-Vide, F. Otto, and H. Fernau, eds.), Lect. Notes Comp. Sci., no. 5196, Springer-Verlag, 2008, pp. 11-27.

[4] M. V. Volkov, Synchronizing automata preserving a chain of partial orders, Theor. Comp. Sci. 410 (2009), 3513 - 3519.

\section{Received: October, 2012}

